\title{
Resection of Lung Cancer with Left Atrium Extension via the Pulmonary Vein: Case Report
}

\author{
Shinjiro Mizuguchi*, Nobuhiro Izumi, Hiroaki Komatsu, Michihito Toda, \\ Kantaro Hara, Toshihiko Shibata, Noritoshi Nishiyama
}

Department of Thoracic Surgery, Osaka City University Hospital, Osaka, Japan

Email: ^m1293795@msic.med.osaka-cu.ac.jp

How to cite this paper: Mizuguchi, S., Izumi, N., Komatsu, H., Toda, M., Hara, K., Shibata, T. and Nishiyama, N. (2018) Resection of Lung Cancer with Left Atrium Extension via the Pulmonary Vein: Case Report. Open Journal of Thoracic Surgery, 8, 13-18.

https://doi.org/10.4236/ojts.2018.81003

Received: February 21, 2018

Accepted: March 26, 2018

Published: March 29, 2018

Copyright $\odot 2018$ by authors and Scientific Research Publishing Inc. This work is licensed under the Creative Commons Attribution International License (CC BY 4.0).

http://creativecommons.org/licenses/by/4.0/

\begin{abstract}
A 49-year-old man had an abnormal shadow on chest X-ray. Enhanced chest computed tomography (CT) revealed an $8-\mathrm{cm}$ diameter right lung mass invading the right chest wall, with a tumor thrombus extending from the superior pulmonary vein into the left atrium. Transesophageal echocardiography confirmed that the tumor adjoined the side wall of the atrium. Endobronchial and CT-guided needle biopsy demonstrated a low-grade carcinoma or small cell carcinoma. Operative findings through left atriotomy under cardiopulmonary bypass showed no tumor invasion of the atrium wall, but protrusion through the pulmonary vein. Frozen sections revealed a non-small cell carcinoma. We performed right upper lobectomy with parietal pleura and mediastinal lymph node dissection after detachment of cardiopulmonary bypass. Pathological examination demonstrated a large-cell neuroendocrine carcinoma p-T4N0M0, stage IIIA. The patient recovered without postoperative complications and tolerated two cycles of adjuvant chemotherapy. He was doing well without symptoms of recurrence 42 months after surgery.
\end{abstract}

\section{Keywords}

Extensive Invasion, Left Atrium, Lung Cancer, Cardiopulmonary Bypass

\section{Introduction}

Approximately $8 \%-10 \%$ of lung cancers invade the heart, especially the left atrium [1], which can lead to widespread systemic embolization and/or outflow tract obstruction. Treatment of these cases is therefore always warranted. However, these T4 patients are generally considered to be inoperable [2], and treatment including radical resection presents a therapeutic challenge. Nevertheless, improvements in operative and perioperative capabilities, including cardiopul- 
monary bypass, dedicated cardiothoracic anesthesia, and treatments for postoperative complications, mean that extended resections for T4N0/1 non-small cell lung cancer (NSCLC) with partial resection of the left atrium have been performed with reasonable postoperative morbidity and mortality [3]. Timely surgical intervention can be life-saving by preventing sudden cardiac arrest due to intracardiac extension of the lung cancer [4].

We present a case of pulmonary large cell neuroendocrine carcinoma complicated with a left atrial tumor thrombus. After careful preoperative evaluation of the tumor thrombus by ultrasonic cardiography, the patient was treated successfully by right upper lobectomy and atriotomy with cardiopulmonary bypass.

\section{Case Report}

A 49-year-old man had an abnormal shadow in the right upper lung field on chest X-ray. He was asymptomatic and had a 75 pack-year smoking history. His physical examination was unremarkable. An enhanced chest computed tomography (CT) scan revealed an 8-cm diameter right lung mass invading the right chest wall, with a tumor thrombus extending from the superior pulmonary vein into the left atrium (Figure 1(a)). Atrial invasion was estimated by transsternal and transesophageal echocardiography, which confirmed that the tumor adjoined the side wall of the atrium and protruded about $3 \mathrm{~cm}$ into the left atrium (Figure 1(b)). A positron emission tomography scan showed no uptake in the mediastinal lymph nodes, but intense ${ }^{18} \mathrm{~F}$-fluorodeoxyglucose uptake was detected by the primary tumor (maximum standardized uptake value (SUV $\left.{ }_{\max } 9.7\right)$, hilum lymph node (SUV $\left.{ }_{\text {max }} 5.6\right)$ and extending atrium tumor $\left(\mathrm{SUV}_{\max } 5.4\right)$. Endobronchial biopsy and CT-guided needle biopsy were suspicious for a low-grade carcinoma or small cell carcinoma. No distant metastasis was found by brain magnetic resonance imaging, abdominal $\mathrm{CT}$, or positron emission tomography-CT.

The case was presented to a multidisciplinary tumor board for consideration of surgical therapy. He was staged as clinical T4N1M0, stage IIIA, but he was considered a suitable candidate for induction chemotherapy followed by surgery because of his age and good performance status. However there were risks of perioperative occult micrometastasis and sudden death. Surgical resection followed by adjuvant chemotherapy was considered as a strategy to reduce the risk of sudden death as a result of systemic tumor embolization due to chemotherapy or cardiac failure. Median sternotomy was performed. Operative findings through left atriotomy under cardiopulmonary bypass showed no invasion of the atrium wall by the tumor, which protruded through the pulmonary vein (PV) 1-3, and PV4-5 was intact (Figure 2). We therefore resected the left atrium tumor while preserving PV4-5, closed PV1-3, and used a bovine pericardium patch for left atrium reconstruction. Although simple upper lobectomy was planned when the tumor was diagnosed as small cell carcinoma, frozen sections revealed a non-small cell carcinoma. Therefore we performed right upper lobectomy with 

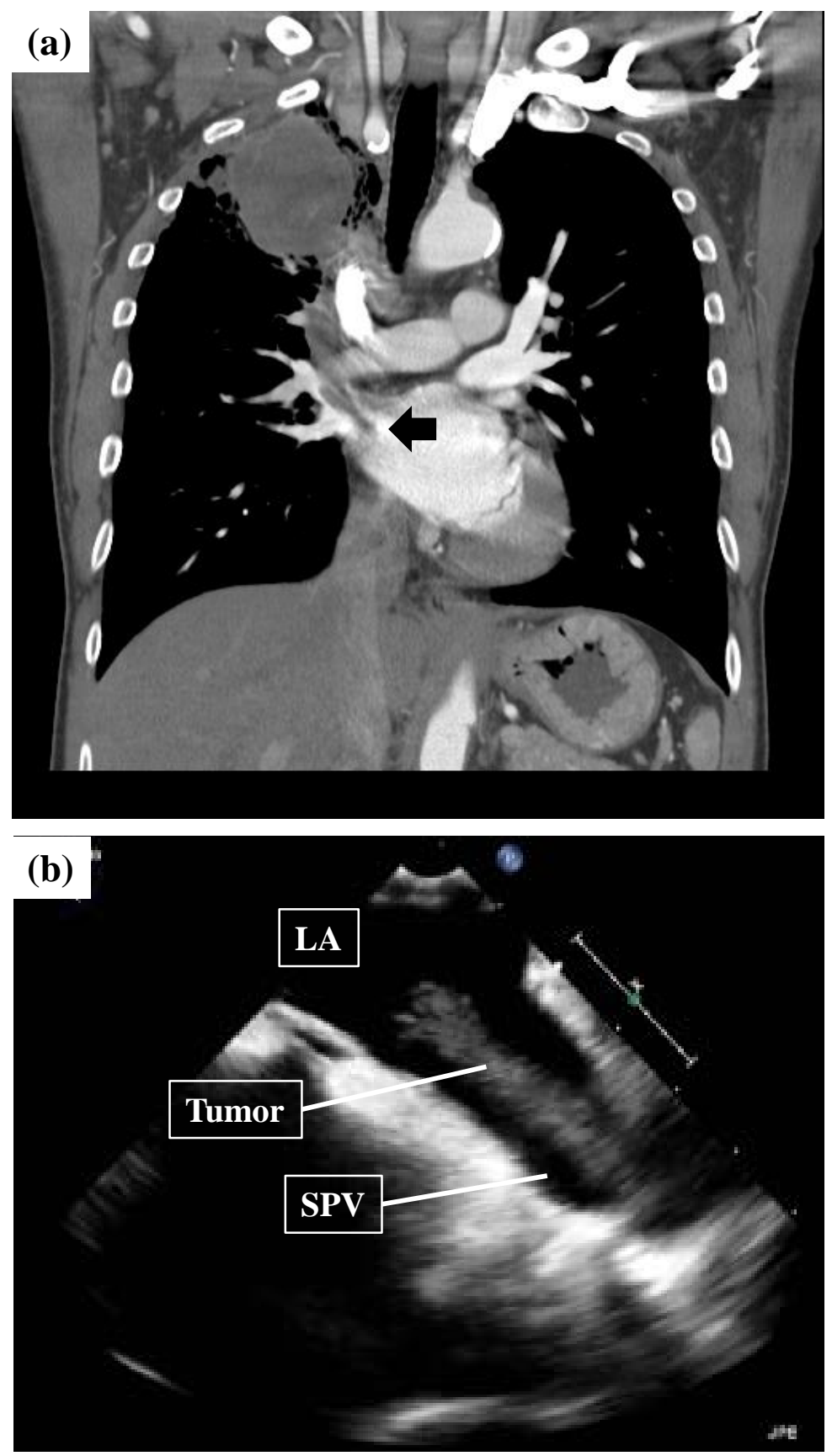

Figure 1. (a) Enhanced thoracic computed tomography scan showing a mass in the right upper lobe with a tumor thrombus extending to the left atrium (dark arrow); (b) Transesophageal echocardiogram showing the tumor protruding into the left atrium and adjoining the side wall, without invasion.

parietal pleura and mediastinal lymph node dissection after detachment of cardiopulmonary bypass.

Pathological examination demonstrated a large cell neuroendocrine carcinoma p-T4N0M0; stage IIIA, without lymph node metastasis. The patient recovered without postoperative complications and received two cycles of adjuvant 


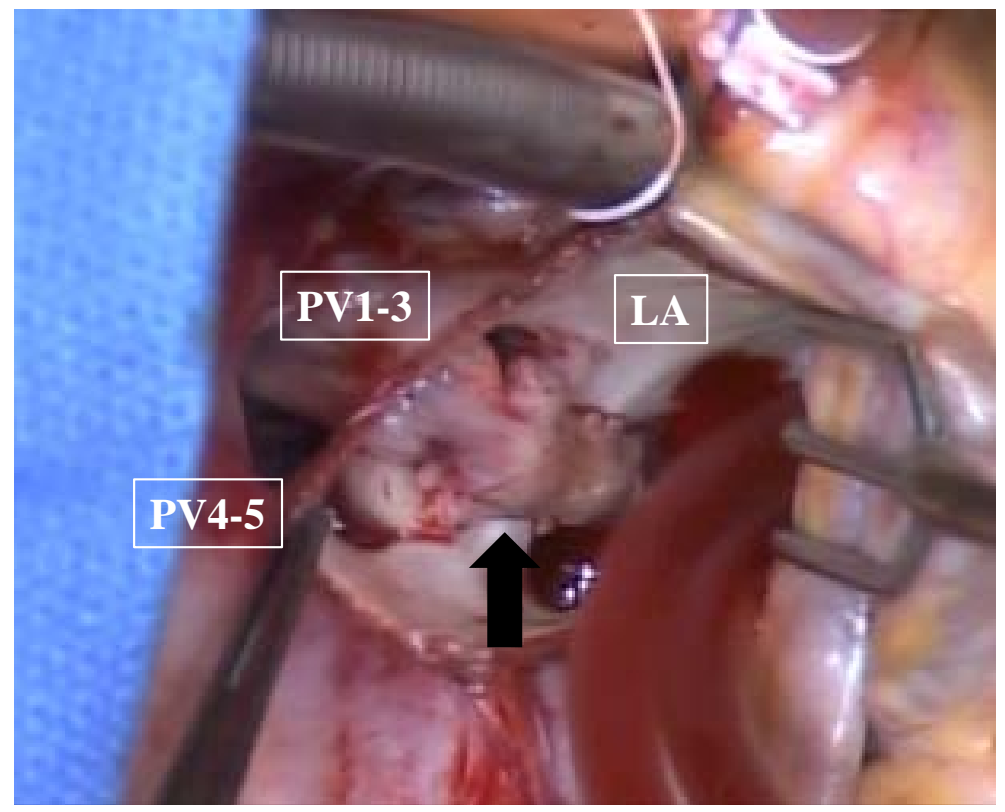

Figure 2. Intraoperative illustration. After incision of the left atrium, the tumor thrombus was exposed with no infiltration into the left atrium or vessel wall. Dark arrow demonstrates the tumor in the left atrium through the pulmonary vein (V1-3). LA, left atrium; PV pulmonary vein.

chemotherapy (irinotecan plus cisplatin), which he tolerated well. He remained well with no symptoms of recurrence 42 months after surgery.

\section{Discussion}

Lung cancer is one of the most common causes of metastatic cardiac tumor, and several cases of intra-cardiac extension via the pulmonary vein have been reported. If left untreated, patients may die from sudden cardiac arrest due to cardia inflow obstruction or massive tumor embolization in the major organs [5] [6], which is one of the most serious complications. Schreffler et al. reported a case of simultaneous acute bilateral lower limb ischemia, and bilateral renal, splenic, and cerebral infarction as a result of multiple emboli originating from invasion of a primary lung malignancy in the left atrium [7]. Given the risk of systemic embolization, mitral obstruction, or sudden death as a result of tumor thrombus detachment, comprehensive preoperative preparation and meticulous intraoperative surgery need to be emphasized to ensure complete resection of the tumor thrombus.

Because of the catastrophic complications of intra-atrial tumors, aggressive treatment is typically pursued regardless of the patient's overall prognosis. Although several studies have demonstrated potentially beneficial palliative effects of stereotactic radiotherapy in patients with stage IV lung cancer invading the left atrium [8] [9], surgical resection remains the historical gold standard treatment. The 5 -year survival rate among 44 patients receiving left atrium resection was $22 \%$, including six cases without distant metastasis who experienced long-term survival over 5 years [10]. In a recent review of 18 previous cases (1995-2013) 
[8], surgery combined with chemotherapy was the most common treatment for this condition, with survival times ranging from 4 - 17 months.

In the current case, we performed surgical resection followed by adjuvant chemotherapy. In another review of nine papers reporting on the postoperative outcomes of patients with primary lung cancer involving the left atrium [11], although the advantages of surgery after induction therapy have not been established for T4 lung cancer involving the left atrium, 25\% (0\% - 60\%) of patients received induction treatment. Importantly, pneumonectomy was selected in $81 \%$ $(63 \%-100 \%)$ of patients and pathological N2 disease was contained in $32 \%$ $(17 \%-53 \%)$. Because recurrences in distant organs were considered in these patients, adjuvant chemotherapy may also play a positive role in the treatment of advanced lung cancer, and pneumonectomy should be avoided to preserve chemotherapy tolerance. Although cardiopulmonary bypass should generally be avoided during the treatment of malignant tumors because of the risk of intraoperative tumor cell dissemination [12], extended lobectomy with left atrium reconstruction under cardiopulmonary bypass, avoiding pneumonectomy, might be appropriate together with adjuvant therapy in patients with left atrium extension NSCLC.

\section{Acknowledgements}

None declared.

\section{Funding}

The authors declare no financial or any other type of support.

\section{Authors' Contributions}

SM, NI, HK, MT, KH and NN actually performed the operation and management of the patient in this case report. TS comprehensively supervised this case report. All authors read and approved the final manuscript.

\section{Consent for Publication}

Consent for publication was obtained from the patients.

\section{Competing Interests}

The authors declare that they have no competing interests.

\section{References}

[1] Spaggiari, L., D’Aiuto, M., Veronesi, G., Pelosi, G., de Pas, T., Catalano, G. and de Braud, F. (2005) Extended Pneumonectomy with Partial Resection of the Left Atrium, without Cardiopulmonary Bypass, for Lung Cancer. The Annals of Thoracic Surgery, 79, 234-240. https://doi.org/10.1016/j.athoracsur.2004.06.100

[2] Ettinger, D.S., Akerley, W., Bepler, G., Blum, M.G., Chang, A., Cheney, R.T., Chirieac, L.R., D’Amico, T.A., Demmy, T.L., Ganti, A.K., et al. (2010) Non-Small Cell Lung Cancer. Journal of the National Comprehensive Cancer Network, 8, 740-801. 
https://doi.org/10.6004/jnccn.2010.0056

[3] Yildizeli, B., Dartevelle, P.G., Fadel, E., Mussot, S. and Chapelier, A. (2008) Results of Primary Surgery with T4 Non-Small Cell Lung Cancer during a 25-Year Period in a Single Center: The Benefit Is Worth the Risk. The Annals of Thoracic Surgery, 86, 1065-1075. https://doi.org/10.1016/j.athoracsur.2008.07.004

[4] Lin, M.T., Ku, S.C., Wu, M.Z. and Yu, C.J. (2008) Intracardiac Extension of Lung Cancer via the Pulmonary Vein. Thorax, 63, 1122.

https://doi.org/10.1136/thx.2007.090373

[5] Kodama, K., Doi, O. and Tatsuta, M. (1990) Unusual Extension of Lung Cancer into the Left Atrium via the Pulmonary Vein. International Surgery, 75, 22-26.

[6] Brandt, R.R., Rubin, J. and Reeder, G.S. (1995) Intracardiac Extension of a Lung Tumor Causing Left Ventricular Inflow Obstruction. Journal of the American Society of Echocardiography, 8, 930-933. https://doi.org/10.1016/S0894-7317(05)80019-7

[7] Schreffler, S.M., Paolo, W.F. and Kloss, B.T. (2012) Spontaneous Showering of Tumor Emboli in a Patient with Advanced Primary Lung Cancer: A Case Report. International Journal of Emergency Medicine, 5, 27.

[8] Li, Y., Lou, J., Qiu, S., Guo, Y. and Pan, M. (2016) Stereotactic Radiotherapy for the Treatment of Lung Cancer with a Giant Left Atrial Tumor Thrombus: A Case Report and Literature Review. Oncology Letters, 11, 2229-2232. https://doi.org/10.3892/ol.2016.4215

[9] Lee, P. and Kishan, A.U. (2012) Radiotherapy Is Effective for a Primary Lung Cancer Invading the Left Atrium. BMJ Case Reports, 2012.

[10] Tsuchiya, R., Asamura, H., Kondo, H., Goya, T. and Naruke, T. (1994) Extended Resection of the Left Atrium, Great Vessels, or Both for Lung Cancer. The Annals of Thoracic Surgery, 57, 960-965. https://doi.org/10.1016/0003-4975(94)90214-3

[11] Tsukioka, T., Takahama, M., Nakajima, R., Kimura, M., Inoue, H. and Yamamoto, R. (2016) Surgical Outcome of Patients with Lung Cancer Involving the Left Atrium. International Journal of Clinical Oncology, 21, 1046-1050. https://doi.org/10.1007/s10147-016-0992-y

[12] Akchurin, R.S., Davidov, M.I., Partigulov, S.A., Brand, J.B., Shiriaev, A.A., Lepilin, M.G. and Dolgov, I.M. (1997) Cardiopulmonary Bypass and Cell-Saver Technique in Combined Oncologic and Cardiovascular Surgery. Artificial Organs, 21, 763-765. https://doi.org/10.1111/j.1525-1594.1997.tb03738.x

\section{Abbreviations}

CT: Computed tomography

NSCLC: non-small cell lung cancer

PET: Positron emission tomography

PV: pulmonary vein

SUV: standardized uptake value 